\title{
Synthesis and assessment of a graphene-based composite photocatalyst
}

Pedro Magalhães, Joana Ângelo, Vera M. Sousa, Olga C. Nunes, Luísa Andrade, Adélio Mendes*

LEPABE - Faculdade de Engenharia, Universidade do Porto, Rua Dr. Roberto Frias, 4200465 Porto, Portugal

\begin{abstract}
A novel composite photocatalyst prepared from graphene and commercial TiO2 (P25 from Evonik) was synthesized, exhibiting enhanced photocatalytic activity for methylene blue degradation, when com-pared with pristine P25. Additionally, the new catalyst showed $20 \%$ more NO conversion under UV light than P25. The band gap of the catalyst, obtained from diffuse reflectance, was $2.95 \mathrm{eV}$ indicating an extended light absorption up to $420 \mathrm{~nm}$. The novel photocatalyst was further tested for inactivating microorganisms showing better results than the reference photocatalyst. Under visible light, the viability loss of the reference bacterial strain Escherichia coli DSM 1103 was two times higher than with the bare P25; it was observed 29\% of inactivation with the P25/graphene composite and $14 \%$ with the P25 sample, following standard ISO 27447:2009.
\end{abstract}

\section{Introduction}

Photocatalysis has attracted the attention of many researchers mainly because it can be used for photoabatement of atmospheric contaminants, water treatment and inactivation of microorganisms both in suspension and on surfaces [1,2]. More recently, photoinactivation of microorganisms has emerged as an alternative disinfection method [3]; especially the use of titanium dioxide in antimicrobial application has been widely discussed $[2,3]$. This special interest on TiO2 material was fueled by the work by Fujishima and Honda [4] in 1972, describing for the first time water splitting using a TiO2 photoelectrode.

Independently of the photocatalytic application, the mechanism behind photocatalysis consists in the generation of electron-hole pairs upon excitation of the photocatalyst with photons showing energy higher than the band gap (in the case of TiO2 ca. $3.2 \mathrm{eV}$ ) -Eq. (1). These holes and electrons can oxidize and reduce surface-adsorbed molecules, respectively. The strong oxidation potential of the photogenerated valence band holes in anatase TiO2 $\left(\mathrm{E}_{\mathrm{VB}}=+3.0 \mathrm{~V}\right.$ vs. normal hydrogen electrode ( $\mathrm{NHE}), \mathrm{pH} 1$ ) originates the formation of hydroxyl radicals $(\mathrm{OH} \bullet)$ when in contact with water - Eq. (2). The reduction potential of anatase TiO2 conduction band electrons is of ca. $0.2 \mathrm{~V}(\mathrm{pH} 1)$ and reduces $\mathrm{O} 2$ to produce superoxide radical (O2•-) --Eq. (3) [5]. These free radicals intermediate the oxidation of organic species at, for example, the surface of microorganisms, originating ultimately their inactivation. 


$$
\begin{aligned}
& \text { Semiconductor } \stackrel{h v}{\rightarrow} \mathrm{e}_{\mathrm{CB}}^{-}+\mathrm{h}_{\mathrm{VB}}^{+} \\
& \text {Semiconductor }\left(\mathrm{h}_{\mathrm{VB}}^{+}\right)+\mathrm{H}_{2} \mathrm{O}_{\text {ads }} \rightarrow \text { Semiconductor }+\mathrm{HO}_{\text {ads }}^{\cdot}+\mathrm{H}^{+}(2) \\
& \text { Semiconductor }\left(\mathrm{e}_{\mathrm{CB}}^{-}\right)+\mathrm{O}_{2 \text { ads }} \rightarrow \text { Semiconductor }+\mathrm{O}_{2}^{--}
\end{aligned}
$$

The extensive use of $\mathrm{TiO} 2$ material as photocatalyst can be explained by its thermal and chemical stability, exhibiting no photocorrosion, readily available, relatively cheap and band edges that are well positioned for producing oxidizing/reducing agents [5]. However, TiO2 absorbs only UV light, preventing its usage under visible spectrum; hence, many efforts have been powered to enhance titanium dioxide photocatalytic activity. Two main actions are being followed: (i) narrowing the semiconductor band gap $\left(E_{g}\right)$; and (ii) decrease the $\mathrm{e}^{-} / \mathrm{h}^{+}$recombination. While the first allows the photocatalyst to absorb a larger fraction of the solar spectrum and eventually reach the visible spectrum, the later allows improving the redox reactions rate at the photocatalyst surface. Several doping techniques have been described targeting the decrease of the semiconductor band gap [6,7]. Even though the doping mechanism is not yet fully understood, the two most used dopants of $\mathrm{TiO} 2$ are $\mathrm{C}$ and $\mathrm{N}$; these dopants act as substitutional anions (substitute oxygen) or interstitial cations (substitute titanium) $[8,9]$. On the other hand, to decrease $\mathrm{e}^{-} / \mathrm{h}^{+}$recombination rate several studies suggest the use of carbon-based supports such as carbon nanotubes (CNTs), fullerene or graphene [8]. These materials have the ability to receive and store photoinjected electrons and thus preventing $\mathrm{e}^{-} / \mathrm{h}^{+}$recombination. In most of the cases, partially oxidized graphene, known as graphene oxide (GO), is used instead of pure graphene due its tunable optical, conductive and chemical properties. Graphene oxide is obtained functionalizing graphene sheets with carboxylic acid, hydroxyl and/or epoxide groups, and thus its properties can be adjusted via chemical modification [10]. The binding of $\mathrm{TiO}_{2}$ to graphene sheets allows improved photo-catalytic performance when compared with their pristine form mainly because: (i) efficient charge separation and transportation;(ii) extended light absorption range; and (iii) enhanced adsorption [11]. TiO2/graphene composites slow the rate of $\mathrm{e}^{-} / \mathrm{h}^{+}$recombination after light excitation [12], increasing the charge transfer rate of electrons. The extended light absorption can be achieved due to doping of $\mathrm{TiO} 2$ with carbon from the graphene, leading to a narrowing of the semiconductor band gap. The enhanced adsorption of the $\mathrm{TiO} 2 /$ graphene composite is mainly attributed to its very large $\pi$-conjugation system and two-dimensional planar structure $[11,13]$. The increase in adsorption may enhance the photocatalytic degradation of methylene blue, assuming the adsorption of reactants is higher than the adsorption of the degradation products. Even though there are some works

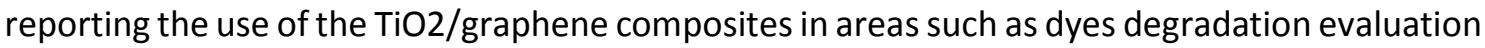
[14] and microorganism photoinactivation [15], the use of TiO2/graphene composites for NO deep oxidation has never been reported in literature.

In this work, a composite photocatalyst was prepared from commercial graphene ( $x G n \mathrm{P}^{\circledR}$ from XG Sciences) and commercial TiO2 (P25 from Evonik) and its performance compared with commercial photocatalysts - P25 and VLP7101 (Kronos). The band-gap of the prepared photocatalyst was assessed by diffuse reflectance and it was tested for methylene blue degradation and NO deep oxidation under UV-light. The photocatalyst was further tested for inactivating microorganisms both under UV radiation and visible light. The viability loss of the reference bacterial strain E. coli DSM 1103 was obtained for both types of radiation and compared with three commercial photocatalysts: P25, VLP7000 and VLP7101. 


\section{Materials and methods}

\subsection{Synthesis of $\mathrm{P} 25 /$ graphene composite photocatalyst}

P25/graphene composite was prepared as described elsewhere [11] with minor modifications. Briefly, oxidized graphene nano-platelets (GNP ox $)$ KMNO4 3:1 graphene nano-platelets (GNP) -were prepared according to a modified Hummer's method. Shortly, $50 \mathrm{~mL}$ of $\mathrm{H}_{2} \mathrm{SO}_{4}$ were added to $2 \mathrm{~g}$ of GNP at room temperature and the solution was cooled using an ice bath, followed by gradual addition of $6 \mathrm{~g}$ of $\mathrm{KMnO}_{4}$. Then, $300 \mathrm{~mL}$ of distilled water was added, followed by addition of $\mathrm{H}_{2} \mathrm{O}_{2}$ until oxygen release stopped. $\mathrm{GNP}_{\text {ox }}$ was washed 5 times with water by centrifugation at $4000 \mathrm{rpm}$ during $15 \mathrm{~min}$. The solid was dispersed in $500 \mathrm{~mL}$ of water by sonication (Bandelin Sonorex R $\mathrm{K} 512 \mathrm{H}$ ) during $5 \mathrm{~h}$ and lyophilized during $72 \mathrm{~h}$. Then, the composite was obtained via a hydrothermal method based on the work by Zhang et al. [11]. Briefly, $2 \mathrm{mg}$ of $\mathrm{GNP}_{\text {ox }}$ was dissolved in a solution of distilled $\mathrm{H}_{2} \mathrm{O}(20 \mathrm{~mL})$ and ethanol $(10 \mathrm{~mL})$ by ultrasonic treatment for $1 \mathrm{~h}$, and $0.2 \mathrm{~g}$ of P25 was added to the obtained GNPox solution and stirred for another $2 \mathrm{~h}$ to get a homogeneous suspension. The suspension was then placed in a $40 \mathrm{~mL}$ Teflon-sealed autoclave and maintained at $120{ }^{\circ} \mathrm{C}$ for $3 \mathrm{~h}$ to simultaneously achieve the reduction of $\mathrm{GO}$ and the deposition of P25 on the carbon support. Finally, the resulting composite was recovered by filtration, rinsed by deionized water several times and dried at room temperature.

The photocatalytic activity of the as-prepared P25/graphene composite was then compared with three different commercial photocatalysts: Aeroxide ${ }^{\circledR}$ TiO2 P25 (Evonik Industries, Germany), Kronos ${ }^{\circledR}$ VLP7000 and VLP7101 (KRONOS Worldwide, Inc., United States of America).

\subsection{Characterization}

\subsubsection{Diffuse reflectance analyses}

Diffuse reflectance spectroscopy is often used to determine the absorption properties of both crystalline and amorphous materials [16]. The band gap of a sample can be obtained from the Tauc equation, which relates the diffuse reflectance and the Kubelka-Munk model to the excitation frequency [16]:

$$
\left(h v F\left(R_{\infty}\right)\right)^{1 / n}=A\left(h v-E_{g}\right)
$$

where, $h$ is the Planck constant, $v$ is the frequency of vibration, $A$ is a constant and $E_{g}$ is the band gap energy.

This equation is obtained multiplying the Kubelka-Munk equation by the energy of the incident radiation $(E=h v)$ and powered to a coefficient $n$, according to the type of the electronic transition of the material. For indirect transitions $n$ equals 2 and for direct transition $n$ is $1 / 2$. Plotting the modified Kubelka-Munk equation as a function of the incident radiation, the band gap of the semiconductor can be obtained extrapolating the linear part of this curve to the $x$ axis, the so-called Tauc plot; the band gap energy is read at the intersection. Diffuse reflectance of the different samples were obtained in a Shimatzu UV-3600 UV-vis-NIR spectrophotometer, equipped with a $150 \mathrm{~mm}$ integrating sphere and using $\mathrm{BaSO}_{4}$ as $100 \%$ reflectance standard. The samples were pressed to form a flat disc that fit into the spectrophotometer sample holder. 


\subsubsection{SEM and XRD analyses}

The morphology and composition of the photocatalysts was obtained from scanning electron microscopy (SEM) coupled with energy dispersive X-ray (EDX) analysis. A FEI Quanta 400FEG ESEM/EDAX Genesis X4 M apparatus equipped with a Schottky field emission gun (for optimal spatial resolution) was used for the characterization of the surface morphology of the photocatalysts with SEM. Images were digitally recorded using a Gatan SC 1000 ORIUS CCD camera (Warrendale, PA, USA). These SEM/EDX analyses were made at Centro de Materiais da Universidade do Porto (CEMUP).

The crystallographic characterization of the samples was per-formed by X-ray diffraction (XRD). The XRD pattern of the selected samples was obtained using a Denchtop X-Ray Diffractometer RIGAKU, model MiniFlex II using Cu X-ray tube (30 KV/15 mA). The data was obtained at $2 \theta$ angles $\left(10-80^{\circ}\right)$, with a step speed of $3.5 \% / \mathrm{min}$. Debye-Scherrer equation was used to determine the crystallite size. The obtained X-ray scans were compared to those of standard database and the phases were assigned comparing with data available in literature.

\subsection{Photoactivity characterization}

The photocatalytic performance of the as prepared semi-conductors was determined by: (a) degradation of dye methylene blue, (b) nitrogen oxide (NO) deep oxidation and (c) photoinactivation of microorganisms. Each of these techniques is described next.

\subsubsection{Methylene blue degradation}

The photodegradation history of methylene blue (MB) dyes was followed by photospectroscopy. In a typical process, aqueous solution of $\mathrm{MB}$ dye $\left(0.01 \mathrm{~g} / \mathrm{L}\right.$, i.e. $\left.2.7 \times 10^{5} \mathrm{M}, 100 \mathrm{~mL}\right)$ and photocatalysts $(50 \mathrm{mg}$ ) were placed in a $250 \mathrm{~mL}$ cylindrical glass vessel. Under ambient conditions and stirring, the photoreactor vessel was exposed to the UV irradiation produced by an ultraviolet (UV-A, highest emission at $365 \mathrm{~nm}$ ) lamp with two $6 \mathrm{~W}$ black-light-blue bulbs (VL206-BLB, Vilbert Lourmat, France); the lamp was positioned $10 \mathrm{~cm}$ away from the vessel (intensity at the catalyst mixture surface was measured with a UV radiometer (HD 2102.2, Delta/OHM, Italy) being $10 \mathrm{Wm}^{-2}$ ). The trial began by putting the mixture during $30 \mathrm{~min}$ in the dark; the mixture was then illuminated for $4 \mathrm{~h}$. Samples were collected every $30 \mathrm{~min}$, centrifuged and the supernatant was analyzed in an UV-vis absorption spectrophotometer (Shimatzu UV3600 UV-vis-NIR spectrophotometer).

\subsubsection{Nitrogen oxide (NO) deep oxidation}

The experimental setup used in NO deep oxidation tests were based on standard ISO 221971:2007 [17]. The experimental setup consists of four main sections: (i) feed, (ii) reactor, (iii) NOx quantification and (iv) computer monitoring/control [18]. Semiconductors were tested as powder films pressed on aluminum slabs with an area of $2 \mathrm{~cm} \times 2 \mathrm{~cm}$. A $50 \%$ of relative humidity feed stream was sup-plied, with a feed rate of $0.7 \mathrm{~L} \mathrm{~min}^{-1}$ of $\mathrm{NO}$ at $1 \mathrm{ppmv}$ in air at $25{ }^{\circ} \mathrm{C}$. The irradiation was provided by a UV lamp (VL-206-BLB, Vilbert Lourmat, France) with an irradiation of $10 \mathrm{Wm}^{-2}$. The photocatalytic activity was assessed from the NO conversion (Eq. (5)): 


$$
X_{\mathrm{NO}}=\left(\frac{C_{\mathrm{NO}}^{\text {in }}-C_{\mathrm{NO}}^{\text {out }}}{C_{\mathrm{NO}}^{\text {in }}}\right) \times 100
$$

where $\mathrm{X}_{\mathrm{NO}}$ is the conversion of NO, $\mathrm{C}_{\mathrm{NO}}$ stands for NO concentration and the superscripts (in and out) refer to the inlet and outlet streams.

\subsubsection{Photoinactivation of microorganisms}

(a) Bacterial strains and culture conditions

E. coli DSM 1103 was used in the photoinactivation trials. E. coli cells were grown overnight (18$20 \mathrm{~h}$ ) at $30{ }^{\circ} \mathrm{C}$ on Plating Count Agar (PCA, LiofilChem, Italy) and the biomass was used to prepare a suspension in sterile saline solution $[0.85 \% \mathrm{NaCl}(\mathrm{w} / \mathrm{v})]$. The cell density of the suspension was adjusted to $106 \mathrm{CFU} \mathrm{mL}{ }^{-1}$ [19] or $103 \mathrm{CFU} \mathrm{mL}^{-1}$ using a calibration curve of optical density versus number of viable cells [colony forming units (CFU) $\mathrm{mL}^{-1}$ ].

(b) Light source and apparatus

A high intensity, long-wave (highest emission at $365 \mathrm{~nm}$ ) ultraviolet (UV-A) lamp with two 6W black-light-blue bulbs (VL- 206- BLB, Vilbert Lourmat, France) was employed on the UV trials. The incident photon flux was $10 \mathrm{Wm}^{-2}$ according to the standard [19]. The visible light trials were conducted with a regular fluorescent light (Philips Master TL-Mini Super 806 W/840) with a light intensity of $50 \mathrm{Wm}^{-2}$.

(c) Experimental procedure for E. coli photoinactivation in suspension

The experimental procedure for determining the E. coli DSM 1103 photoinactivation is described elsewhere [20]. Briefly, the photocatalyst (P25, P25/graphene, VLP7000 or VLP7101) was added to the cell suspension at a concentration of $0.2 \%(\mathrm{w} / \mathrm{v})$. For each photocatalyst two initial cellular

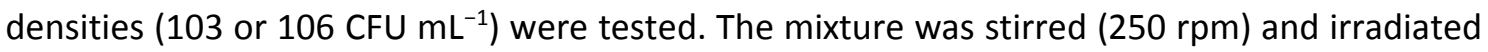
with UV radiation or visible light for $40 \mathrm{~min}$. After this time, the mixture was kept in the dark for 20 min for ensuring that the photocatalyst had no activity, which usually is still observed for a few minutes after being irradiated [21].

At the initial ( $\left.t_{i}=0 \mathrm{~min}\right)$ and final time $\left(t_{f}=40+20 \mathrm{~min}\right)$, the cell suspension was serially diluted in sterile saline solution and aliquots of $100 \mu \mathrm{L}$ were spread on PCA, as described in Koch [22]. After incubation at $30{ }^{\circ} \mathrm{C}$ for $24 \mathrm{~h}$, the colony forming units, corresponding to the viable cells, were enumerated in plates containing 30-300 CFU. The photoinactivation fraction (viability loss) and log reduction were determined as shown in Eq. (6) and Eq. (7), respectively:

$$
\begin{aligned}
& \text { Viability } \operatorname{loss}(\%)=\left(\frac{M_{\mathrm{i}}-M_{\mathrm{f}}}{M_{\mathrm{i}}}\right) \times 100 \\
& \text { Log reduction }=\log M_{\mathrm{i}}-\log M_{\mathrm{f}}
\end{aligned}
$$

where $M_{i}$ and $M_{f}$ are the initial and final E. coli viable counts, respectively. 
(d) Controls

Control assays in the dark, under UV radiation and under visible light in the absence of photocatalyst were performed to assess the influence of the experimental conditions and of the irradiation on cell inactivation, respectively. All the assays were performed in triplicate, inside a clean chamber.

\section{Results and discussion}

\subsection{Photocatalysts characterization}

The band gaps of the three photocatalysts used in this work -P25, VLP7101 and P25/graphene - were calculated from the diffuse reflectance spectra (Fig. 1). The band gap obtained for the composite photocatalyst was $2.95 \mathrm{eV}$, whilst the values obtained for commercial P25 were 3.03 $\mathrm{eV}$ and $3.16 \mathrm{eV}$, corresponding to the two different crystalline forms of titanium dioxide respectively, rutile and anatase, and for VLP7101 was $2.75 \mathrm{eV}$. The slight decrease in the band gap between P25/graphene and P25 may be attributed to some doping of TiO2 by the carbon molecules of graphene [11]. The specific mechanism of carbon doping is not yet fully understood. However, several authors reported that carbon doping leads to a band gap narrowing, causing a red-shift in the absorption spectrum of the photocatalyst [8]. Carbon dopant can be a substitutional anion (substitutes oxygen) or an interstitial cation (substitutes titanium). The substitutional anion behavior of carbon is related to its -4 oxidation state in Ti-C bond in carbides, and the interstitial cation behavior is related to its +4 state in the $\mathrm{C}-\mathrm{O}$ bond in carbonates [8]; decreases in the $\mathrm{TiO} 2$ band gap originated by carbon doping can range from 0.1 $\mathrm{eV}$ to $1.05 \mathrm{eV}$ [8]. However, there is a threshold for dopant concentration at around $2 \%$ of the photocatalyst mass; high dopant concentration leads to a dopant segregation phase [23]. Furthermore, the enhanced photocatalytic activity of VLP7101 can also be ascribed to the presence of an organic sensitizer, as it was already reported [24].

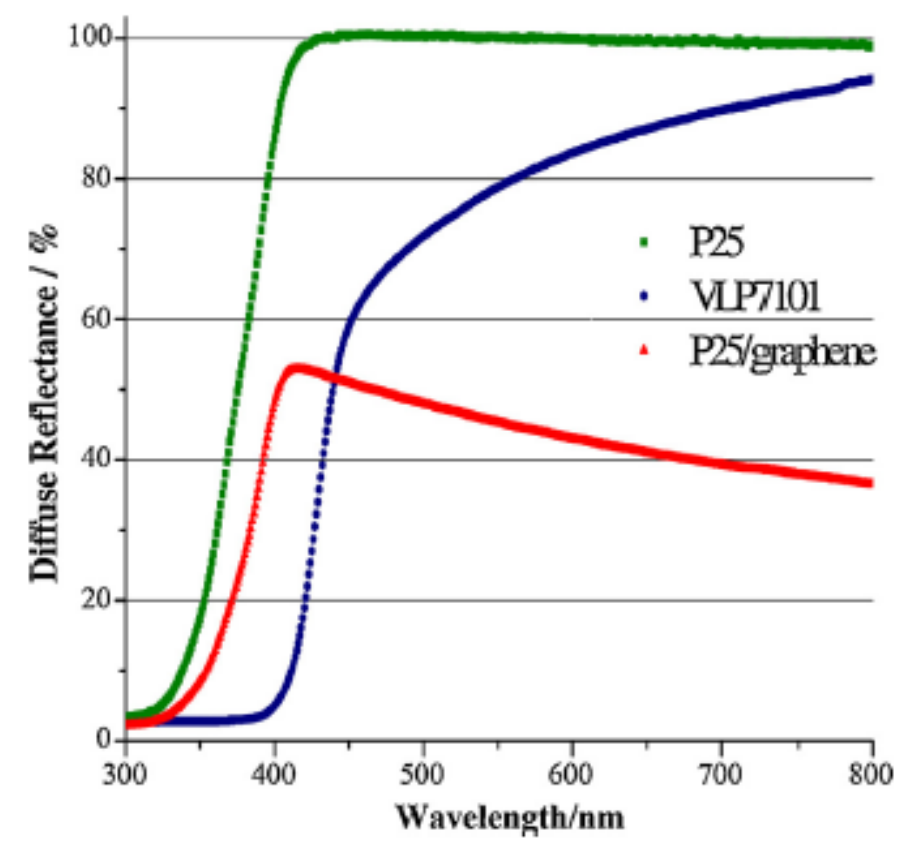

Fig. 1. Diffuse reflectance spectra of VLP7101, P25 and P25/graphene photocatalysts 
XRD analyses were performed to assess the crystallinity of the photocatalysts - Fig. 2. XRD allows determining the crystalline structure of the photocatalysts and, in some cases, to determine the crystal size.

The composite photocatalyst showed similar XRD pattern when compared to P25. Furthermore, no diffraction peaks for carbon species were observed in the composite, which might be due to its low amount and relatively low diffraction intensity of graphene. Furthermore, the similarity of patterns between P25 and the P25/graphene composite evidences that crystallinity, crystal size and modifications on the crystal structure of $\mathrm{TiO} 2$ are not responsible for differences between the photocatalytic activity of these two photocatalysts. It is also possible to observe that VLP7101 is only anatase, contrarily to P25 that is composed by anatase and rutile. Even though it is believed that the interaction between anatase and rutile phases (80/20 wt.\%) improve the photoactivity of P25 [25], VLP7101 present normally higher photoactivity than P25 [18]; this higher catalytic activity was ascribed to an aromatic carbon compound photo sensitizer, as reported elsewhere [24].

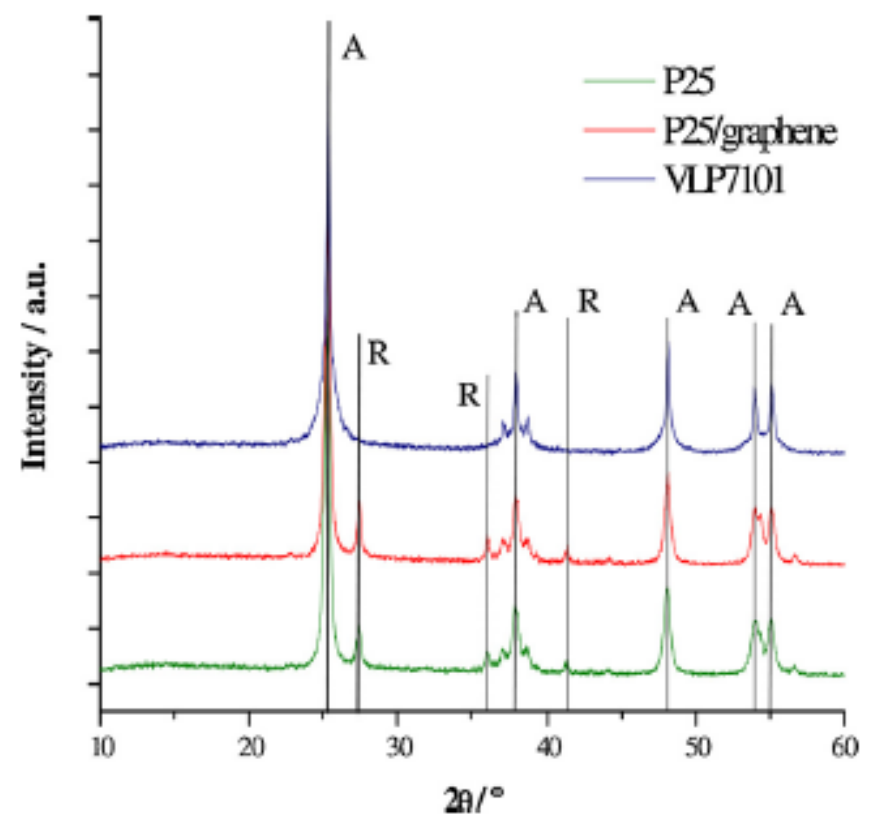

Fig. 2. XRD patterns of VLP7101, P25 and P25/graphene photocatalysts. 
SEM images of P25 and VLP7101 are presented in Figs. 3 and 4.

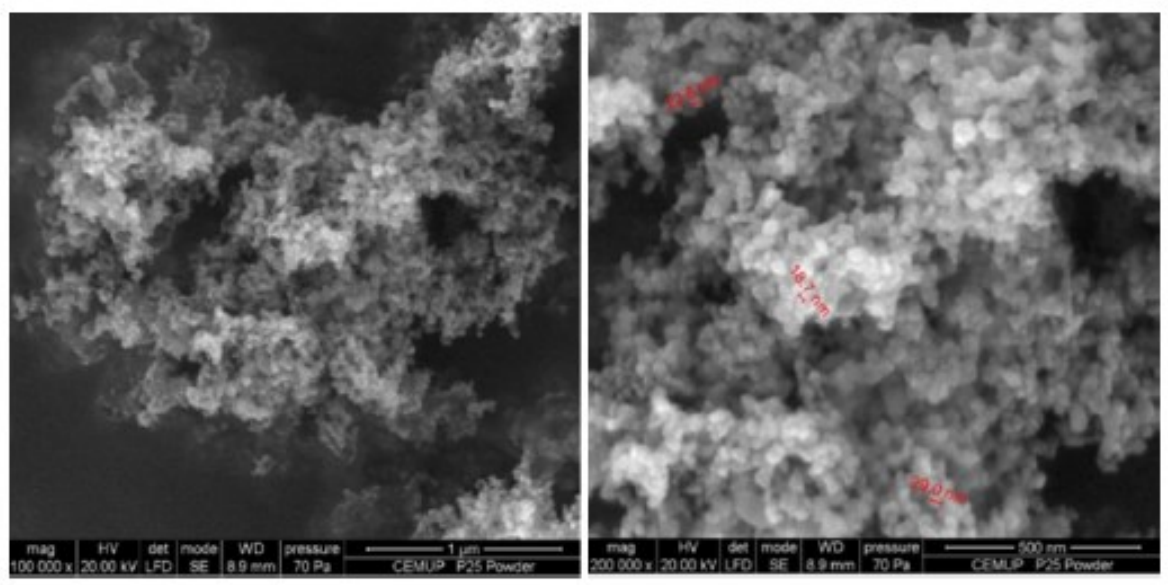

Fig. 3. SEM images of $P 25$.
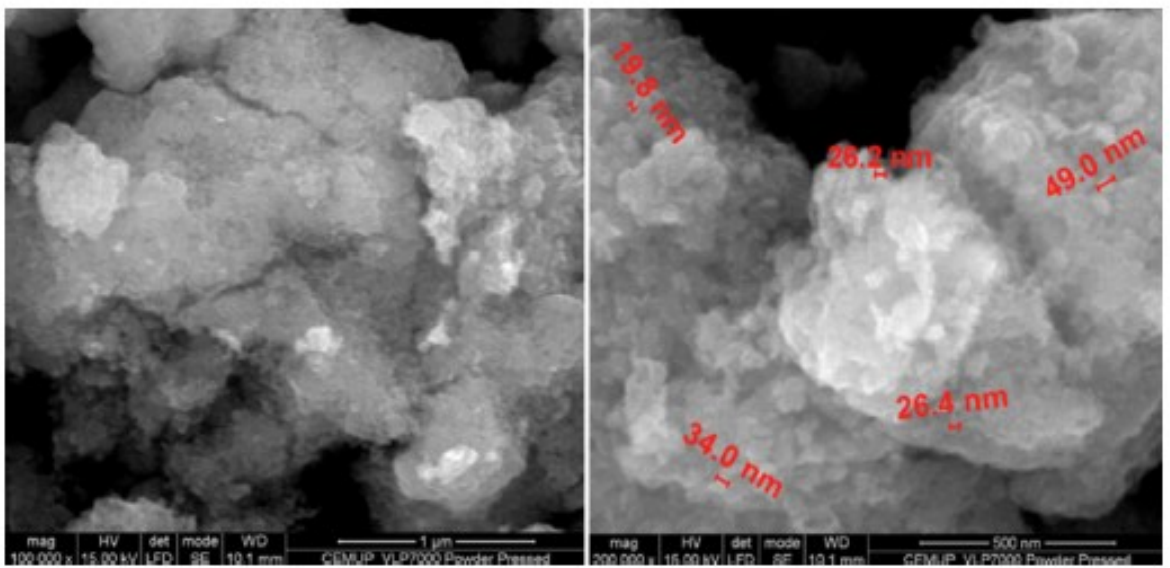

Fig. 4. SEM images of VLP7101.

Fig. 3 shows the morphology of P25 sample. The high agglomeration observed is caused by the high surface energy of the nanoparticles [26]. As it can be seen, the primary particles present sizes in nanometer range. These particles agglomerate forming aggregates with different shapes and morphologies, which influence the surface roughness, haze and transparency of the bulk material [26]. The approximate size of the P25 nanoparticles is between 15 and $30 \mathrm{~nm}$, which is in accordance with values presented in literature [27].

Fig. 4 shows two SEM images of VLP7101 photocatalyst. The particles have a size of 15-50 nm and form also large agglomerates. P25/graphene composite, as already shown previously [11], is composed by P25 nanoparticles deposited on the graphene sheets. These particles bond preferably to the wrinkles and edges of the graphene nanoplatelets, initially covered with carboxylic acid groups. These carboxylic acid groups interact with the hydroxyl groups forming chemical P25/graphene bonds [11]. 


\subsection{Photoactivity characterization}

The photoactivity of the composite photocatalyst, P25 and VLP7101 was assessed based on the methylene blue degradation history and NO photo-conversion. Regarding the methylene blue degradation under UV radiation it is possible to observe in Fig. 5 an increase in the activity of the composite photocatalyst when compared with commercial P25. Comparing the synthesized photocatalyst with VLP7101, both showed similar activities.

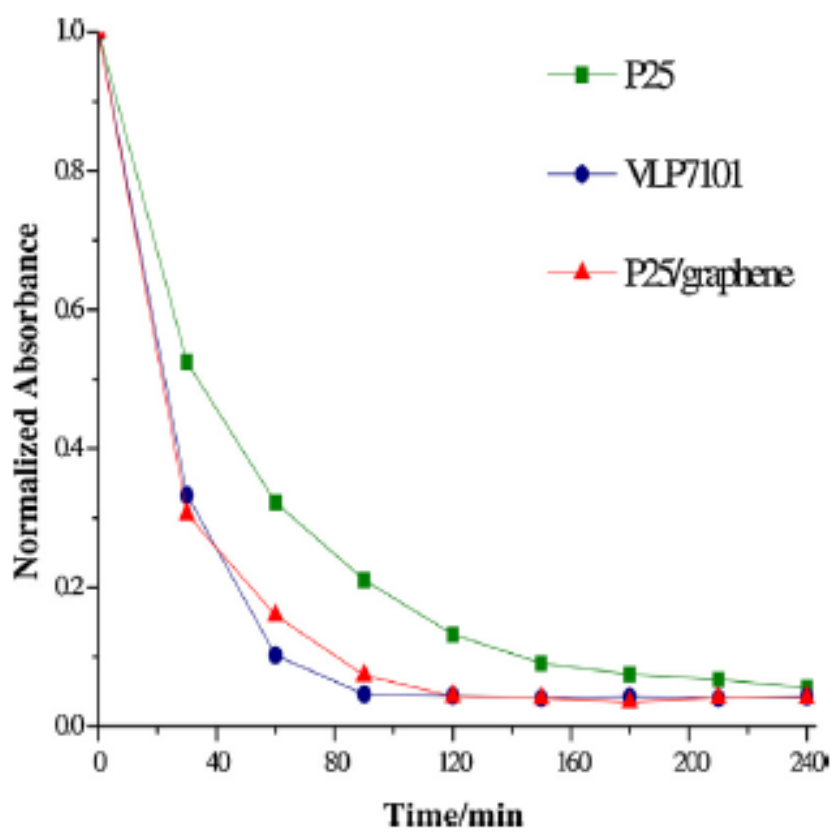

Fig. 5. Normalized absorbance values of methylene blue degradation during 240 min (lines were added to improve the readability) for P25 and VLP70101 commercial photocatalysts and P25/graphene photocatalyst

The increased photocatalytic activity of the P25/graphene composite to the methylene blue degradation compared to pristine P25 should be ascribed to the decrease of $\mathrm{e}^{-} / \mathrm{h}^{+}$ recombination, which is related to the ability of graphene to accept photogenerated electrons by P25 [11], and to an increased adsorption of methylene blue in the support graphene sheets. Actually, graphene support has a much higher adsorption capacity compared with P25 particles. For the relevant methylene blue concentrations, the equilibrium adsorbed concentration on P25 was 4 orders of magnitude smaller than on the graphene used, while on P25/graphene composite was 2 orders of magnitude smaller. This points out for the critical role of the graphene support in the P25/graphene photocatalyst concerning the methylene blue adsorption.

Nguyen-Phan et al. [28] reported the preparation of TiO2/graphene composites with enhanced adsorption and photocatalytic activity under both UV and visible radiation. These authors state that the increase in graphene content, from 1 to $10 \mathrm{wt} . \%$, facilitates the photodegradation rate of methylene blue. The greater photocatalytic performance of TiO2/graphene composite was attributed to the formation of both $\pi-\pi$ conjugations between dye molecules and aromatic rings and to ionic interactions between methylene blue and oxygen-containing functional groups at the edges or on the surfaces of carbon-based nanosheets. Graphene oxide works as adsorbent 
for the methylene blue, electron acceptor and photosensitizer originating an enhanced dye photodecomposition.

Methylene blue degradation is a technique widely used to assess the photocatalytic activity due to its simplicity and easy use. However, this relevant standard [29] presents limitations. This standard assumes the use of methylene blue with very high purity, which is not always possible to obtain. The $\mathrm{pH}$ of the solution should be defined as it influences the adsorption of methylene blue on titanium dioxide. Finally, the standard is mainly applicable to low active photocatalytic films due to problems of stirring and diffusion, among others [30]. Standard ISO 22197-1:2007 [17] uses NO photooxidation to infer about the photoactivity. Since NO is gas, the mass transfer limitations associated to the methylene blue method do not hold here. Because that, this standard is now recommended though its higher complexity. Fig. 6 shows NO conversion history catalyzed by P25, VLP7101 and P25/graphene, under UV light.

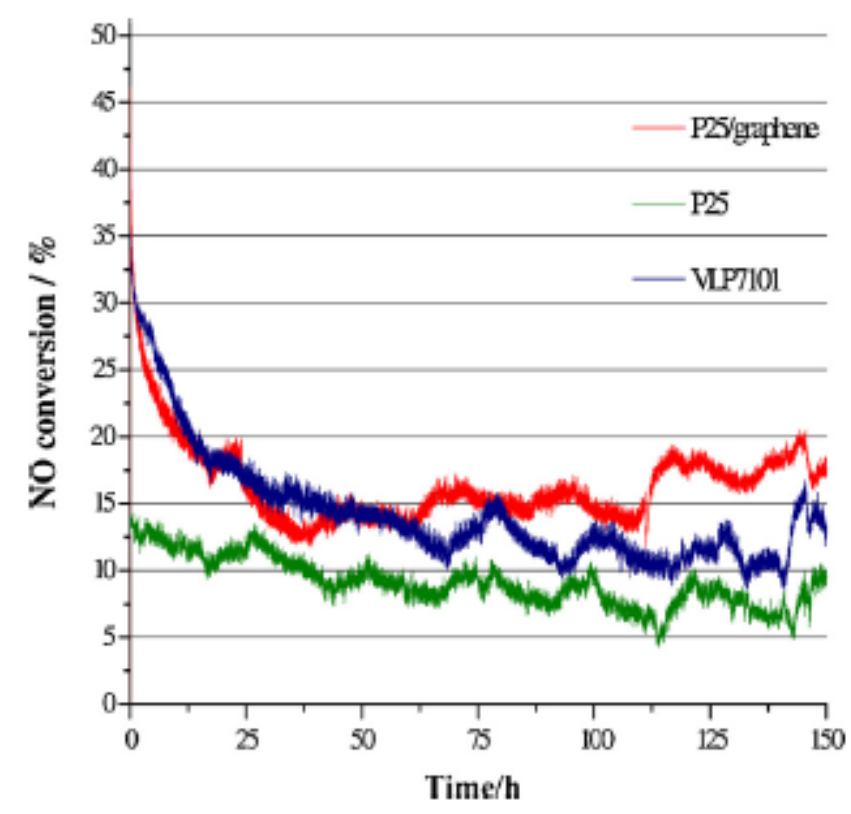

Fig. 6. NO conversion for $\mathrm{P} 25$ and $\mathrm{P} 25 /$ graphene composite during $150 \mathrm{~h}$ under UV radiation.

The prepared photocatalyst presented higher initial and steady state NO conversion than P25 and VLP7101. Furthermore, after the first $24 \mathrm{~h}$, the prepared photocatalyst showed good stability throughout the $150 \mathrm{~h}$. This increased conversion can be ascribed to several factors that were already discussed before. P25/graphene composite slows $\mathrm{e}^{-} / \mathrm{h}^{+}$recombination rate since graphene support increases the draining rate of electrons. Then, the extended light absorption of P25/graphene, supported by a smaller band gap ( $2.95 \mathrm{eV}$ ), allows the absorption of a broader range of wavelengths.

Finally, aiming for a possible future commercial application of the composite photocatalyst, photoinactivation trials were conducted. The initial value of cellular density used was chosen to be around $1 \times 10^{6} \mathrm{CFU} \mathrm{mL}^{-1}$, based on standard ISO 27447:2009 used for evaluating the antibacterial activity of semiconducting photocatalytic materials [19]. Moreover, the order of magnitude $10^{6}$ is also referred to in the standard ISO 11137-2:2013, applied to assess the efficiency of a given method on the sterilization of health care products [31]. 
Under dark conditions the cell inactivation values in the pres-ence of P25 and P25/graphene composite were, as expected, low (around 6\% and $8 \%$, respectively) and similar to those obtained in the absence of photocatalyst (around 5\%), showing that these photocatalysts do not inhibit $E$. coli in the absence of irradia-tion - Fig. 7 and Fig. S1. Contrarily to what was expected, under the same conditions, the cell inactivation values for the carbon-modified VLP7000 and VLP7101 ranged from $\sim 5$ to $65 \%$ for assays carried out over one year. These results show that VLP7000 and VLP7101 present some toxicity towards E. coli cells in the absence of irradiation. Additionally, these values also point out that these photocatalysts did not present stability, as it was already reported in previous studies [24,32]. Due to the mutagenic effect of UV irradiation, cell inactivation under UV was approximately 51\% - Fig. 7 and Fig. S1.
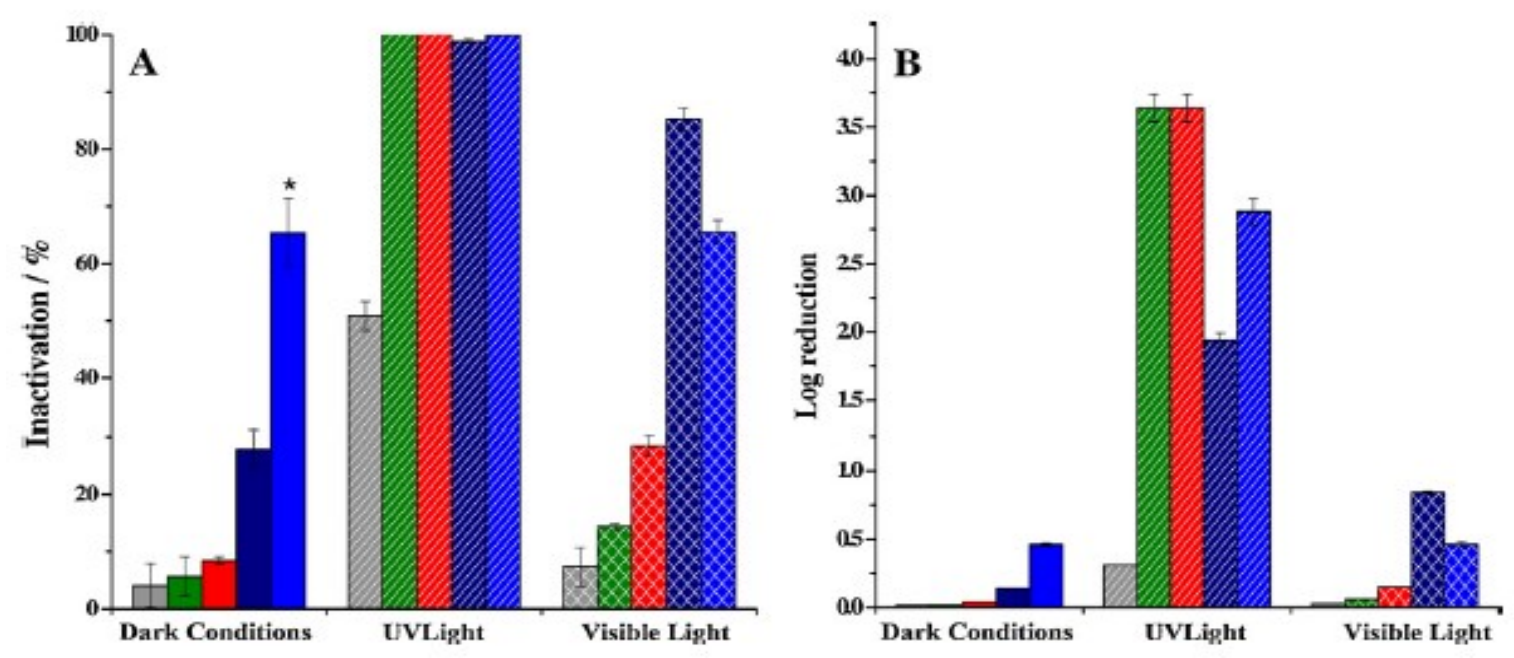

Fig. 7. E. coli viability loss (A) and log reduction (B) under dark, UV radiation and visible light for P25 (green), P25/graphene (red), VLP7101 (dark blue), VLP7000 (light blue) and without photocatalyst (grey). Results are mean values $(n=3)$ and the error bars represent the standard

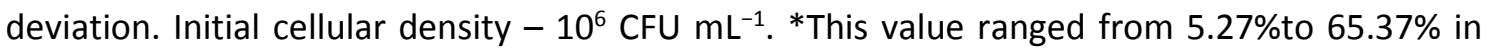
trials conducted over a year.

Higher cell viability losses were obtained in the UV-photoinactivation trials. Under these conditions, similar cell inactivation values $(99.98 \%)$, corresponding to log reduction values of around 3.6, were obtained for P25 and P25/graphene composite. Comparatively lower cell inactivation values were observed when VLP7000 and VLP7101 were irradiated with UV $199.87 \%$ and $98.85 \%$, respectively), corresponding to log reduction values of 2.8 and 1.9 , respectively.

Under visible light, the prepared P25/graphene composite produced two times higher viability loss values ( 29\%), when com-pared with the commercial P25 ( 14\%). Under the same conditions, cell inactivation with VLP7000 and VLP 7101 was much higher ( $65 \%$ and $85 \%$, respectively) than the ones obtained for the produced P25/graphene ( $29 \%)$. However, as mentioned above, these commercial photocatalysts did not present stability, promoting cell inhibition even under dark conditions.

The influence of the initial cellular density on the photoinactivation was also studied. Additional trials carried out with P25 and the P25/graphene were conducted with an initial cellular density

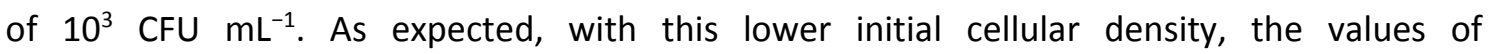
photoinactivation obtained were higher than those obtained with an initial cellular density of 
$10^{6} \mathrm{CFU} \mathrm{mL} \mathrm{mL}^{-1}-$ Fig. 8 and Fig. S2. Total inactivation (log reduction value of 3) was verified when both catalysts were irradiated with UV. Under visible light, viability loss values reached around $15 \%$ for $\mathrm{P} 25$ and $33 \%$ for the $\mathrm{P} 25 /$ graphene composite. Although, the log reduction values were still low (0.07 and 0.18 for P25 and P25/graphene composite, respectively), the increase in the photoinactivation efficiency of the prepared composite can be ascribed to its slower rate of $\mathrm{e}^{-} / \mathrm{h}^{+}$ recombination and extended light absorption range, as suggested by its lower band gap value when compared with that of P25.
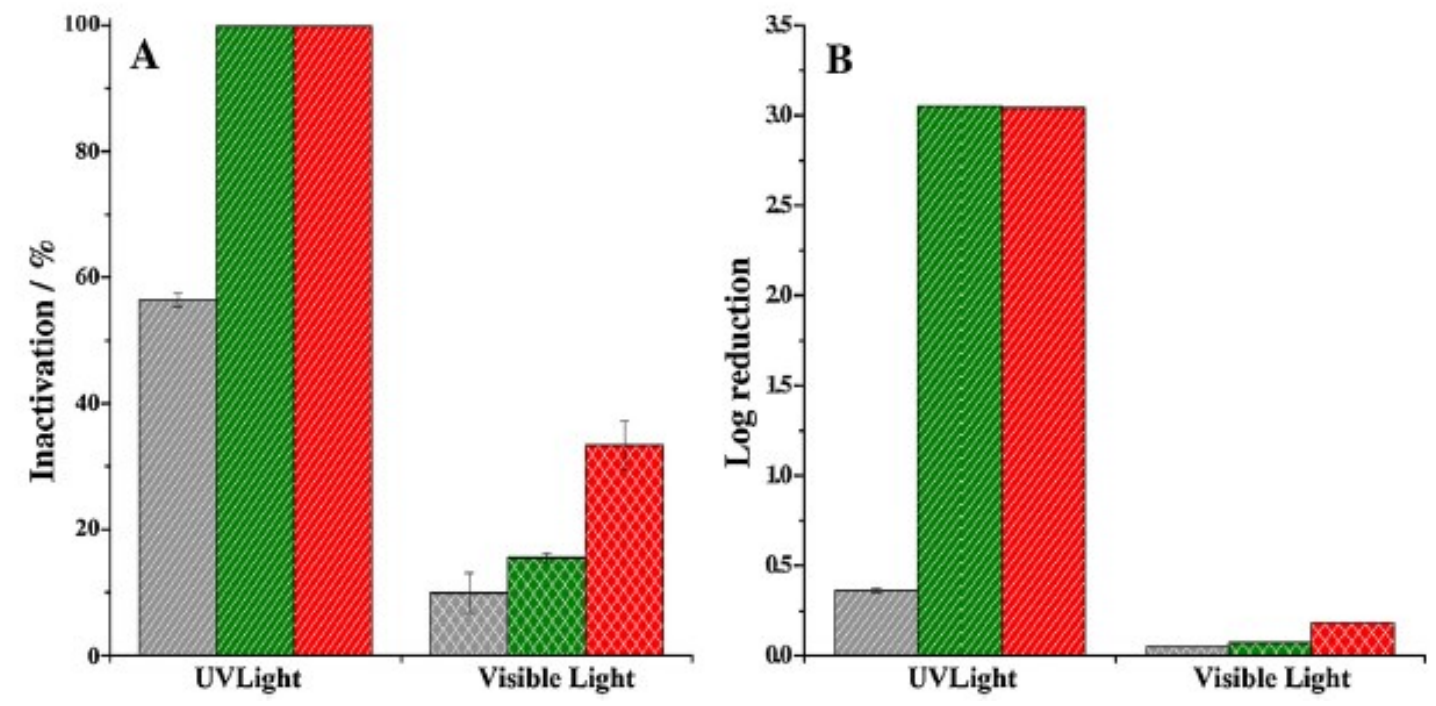

Fig. 8. E. coli viability loss (A) and log reduction (B) under UV radiation and visible light for $P 25$ (green), P25/graphene (red) and without the use of a photocatalyst (grey). Results are mean values $(n=3)$ and the error bars represent the standard deviation. Initial cellular density $-10^{3}$ $\mathrm{CFU} \mathrm{mL}^{-1}$.

\section{Conclusion}

The prepared $\mathrm{TiO}_{2} /$ graphene photocatalyst presented better results for methylene blue and NO oxidation under UV radiation when compared with P25. Additionally, regarding photoinactivation of $E$. coli DSM 1103, the new photocatalyst exhibited higher inactivation activity under visible light than the commercial P25. When compared with VPL7101, the prepared photocatalyst showed similar photoactivity for methylene blue degradation and higher photoactivity regarding the NO oxidation. Both VLP7101 and VLP7000 achieved higher values of E. coli DSM 1103 inactivation than the prepared TiO2/graphene. However, it was observed that VLP7101 and VLP7000 showed high values of inactivation under dark conditions, suggesting toxic properties of these materials.

Further developments in photoinactivation under visible light using TiO2/graphene composites are expected not only from the improvement of this material but also from the use of suitable co-catalysts. 


\section{Acknowledgements}

Pedro Magalhães and Joana Ângelo are grateful to the Portuguese Foundation for Science and Technology (FCT) for their Ph.D. grants (ref: SFRH/BD/78827/2011 and ref: SFRH/BD/79974/2011, respectively). Luísa Andrade acknowledges European Research Council for funding within project BI-DSC - Building Integrated Dye sensitized Solar Cells (Contract Number: 321315). The research was supported by FCT, projects PTDC/EQU-EQU/115614/2009 and FCT-CAPES cooperation 2013-2014.

\section{Appendix A. Supplementary data}

Supplementary data associated with this article can be found, in the online version, at http://dx.doi.org/10.1016/j.bej.2015.05.016

\section{References}

[1] J. Colls, Air Pollution, Spon Press, 2002.

[2] H.A. Foster, I.B. Ditta, S. Varghese, A. Steele, Photocatalytic disinfection using titanium dioxide: spectrum and mechanism of antimicrobial activity, Appl. Microbiol. Biotechnol. 90 (2011) 1847-1868.

[3] T. Matsunaga, R. Tomoda, T. Nakajima, H. Wake, Photoelectrochemical sterilization of microbial cells by semiconductor powders, FEMS Microbiol. Lett. 29 (1985) 211-214.

[4] A. Fujishima, K. Honda, Electrochemical photolysis of water at a semiconductor electrode, Nature 238 (1972) 37-38.

[5] A. Fujishima, T.N. Rao, D.A. Tryk, Titanium dioxide photocatalysis, J. Photochem. Photobiol. C 1 (2000) 1-21.

[6] S. Banerjee, S.C. Pillai, P. Falaras, K.E. O'shea, J.A. Byrne, D.D. Dionysiou, New insights into the mechanism of visible light photocatalysis, J. Phys. Chem. Lett. 5 (2014) 2543-2554.

[7] P. Magalhães, L. Andrade, V.M. Sousa, O.C. Nunes, A. Mendes, Titanium dioxide photocatalysis: fundamentals and application on photoinactivation, Chem. Eng. J. (2014).

[8] R. Leary, A. Westwood, Carbonaceous nanomaterials for the enhancement of TiO2 photocatalysis, Carbon 49 (2011) 741-772.

[9] B. Viswanathan, K.R. Krishanmurthy, Nitrogen incorporation in TiO2: does it make a visible light photo-active material? Int. J. Photoenergy 2012 (2012) 10.

[10] Q. Xiang, J. Yu, M. Jaroniec, Graphene-based semiconductor photocatalysts, Chem. Soc. Rev. 41 (2012) 782-796.

[11] H. Zhang, X. LV, Y. Li, Y. Wang, J. Li, P25-graphene composite as a high performance photocatalyst, ACS Nano 4 (2010) 380-386.

[12] G. Williams, B. Seger, P.V. Kamat, TiO2-graphene nanocomposites. UV-assisted photocatalytic reduction of graphene oxide, ACS Nano 2 (2008) 1487-1491. 
[13] N.T. Khoa, M.W. Pyun, D.H. Yoo, S.W. Kim, J.Y. Leem, E.J. Kim, S.H. Hahn, Photodecomposition effects of graphene oxide coated on $\mathrm{TiO} 2$ thin film prepared by electronbeam evaporation method, Thin Solid Films 520 (2012) 5417-5420.

[14] K. Zhou, Y. Zhu, X. Yang, X. Jiang, C. Li, Preparation of graphene-TiO2 composites with enhanced photocatalytic activity, New J. Chem. 35 (2011) 353-359.

[15] O. Akhavan, E. Ghaderi, Photocatalytic reduction of graphene oxide nanosheets on TiO2 thin film for photoinactivation of bacteria in solar light irradiation, J. Phys. Chem. C 113 (2009) 20214-20220.

[16] A.B. Murphy, Band-gap determination from diffuse reflectance measurements of semiconductor films, and application to photoelectrochemical water-splitting, Sol. Energy Mater. Sol. Cells 91 (2007) 1326-1337.

[17] ISO 22197-1:2007 Fine ceramics (advanced ceramics, advanced technical ceramics) Test method for air-purification performance of semiconducting photocatalytic materials - Part 1: Removal of nitric oxide, ISO (2007).

[18] C. Águia, J. Ângelo, L.M. Madeira, A. Mendes, Photo-oxidation of NO using an exterior paint - screening of various commercial titania in powder pressed and paint films, J. Environ. Manage. 92 (2011) 1724-1732.

[19] ISO 27447:2009 Fine ceramics (advanced ceramics, advanced technical ceramics) - Test method for antibacterial activity of semiconducting photocatalytic materials, ISO (2009).

[20] V.M. Sousa, C.M. Manaia, A. Mendes, O.C. Nunes, Photoinactivation of various antibiotic resistant strains of E. coli using a paint coat, J. Photochem. Photobiol. A 251 (2013) 148-153.

[21] Z. Huang, P.-C. Maness, D.M. Blake, E.J. Wolfrum, S.L. Smolinski, W.A. Jacoby, Bactericidal mode of titanium dioxide photocatalysis, J. Photochem. Photobiol. A 130 (2000) 163-170.

[22] A.L. Koch, Growth measurement, in: R.G.E.M.P. Gerhardt, W.A. Wood, N.R. Krieg (Eds.), Methods for General and Molecular Bacteriology, ASM, Press, Washington DC, 1994, pp. 248292.

[23] R. Van De Krol, M. Grätzel, Photoelectrochemical Hydrogen Production, Springer, 2011, pp. 13-67.

[24] P. Zabek, J. Eberl, H. Kisch, On the origin of visible light activity in carbon-modified titania, Photochem. Photobiol. Sci. 8 (2009) 264-269.

[25] M. Pelaez, N.T. Nolan, S.C. Pillai, M.K. Seery, P. Falaras, A.G. Kontos, P.S.M. Dunlop, J.W.J. Hamilton, J.A. Byrne, K. O'Shea, M.H. Entezari, D.D. Dionysiou, A review on the visible light active titanium dioxide photocatalysts for environmental applications, Appl. Catal. B Environ. 125 (2012) 331-349.

[26] N. Mandzy, E. Grulke, T. Druffel, Breakage of TiO2 agglomerates in electrostatically stabilized aqueous dispersions, Powder Technol. 160 (2005) 121-126.

[27] B. Ohtani, O.O. Prieto-Mahaney, D. Li, R. Abe, What is Degussa (Evonik) P25?Crystalline composition analysis, reconstruction from isolated pure particles and photocatalytic activity test, J. Photochem. Photobiol. A 216 (2010) 179-182. 
[28] T.-D. Nguyen-Phan, V.H. Pham, E.W. Shin, H.-D. Pham, S. Kim, J.S. Chung, E.J. Kim, S.H. Hur, The role of graphene oxide content on the adsorption-enhanced photocatalysis of titanium dioxide/graphene oxide composites, Chem. Eng. J. 170 (2011) 226-232.

[29] ISO 10678: 2010, Fine Ceramics, Advanced Technical Ceramics -Determination of Photocatalytic Activity of Surfaces in an Aqueous Medium by Degradation of Methylene Blue, ISO, Geneva, 2010.

[30] A. Mills, C. Hill, P.K.J. Robertson, Overview of the current ISO tests for photocatalytic materials, J. Photochem. Photobiol. A 237 (2012) 7-23.

[31] ISO 1137-2:2013, Sterilization of Health Care Products - Radiation, ISO, Geneva, 2010.

[32] R. Quesada-Cabrera, A. Mills, C. O'Rourke, Action spectra of P25 TiO2 and a visible light absorbing, carbon-modified titania in the photocatalytic degradation of stearic acid, Appl. Catal. B Environ. 150-151 (2014) 338-344. 